\title{
Hypocaloric Peripheral Parenteral Nutrition Administered in Intestinal Reconnection Postoperative Patients with 3-5 Days Oral Fasting Indicated: A Case Report
}

\author{
Jesus Manuel De Aldecoa-Castillo Tamara Daniela Frydman Daniela Rubio-Mendoza \\ Carlos Alvarado-Vargas Melchor Alpízar-Salazar \\ Centro Especializado en Diabetes, Obesidad, Prevención y Enfermedades Cardiovasculares, Mexico City, Mexico
}

\section{Keywords}

Parenteral nutrition · Colorectal neoplasms · General surgery

\begin{abstract}
Postoperative patients of intestinal reconnection in Morelia, Mexi$\mathrm{co}$, are usually in for 3-5 days of oral fast, which increases protein catabolism in the patient, thus lengthening their hospital stay and increasing the risk of developing metabolic comorbidities. Hypocaloric peripheral parenteral nutrition (HPPN) reduces proteolysis and improves inflammatory markers in these patients. The aim of this case report is to determine whether or not peripheral parenteral nutrition (PPN) improves inflammation, lessening the postoperative risk. A 62-year-old female patient and her cancer diagnosis
\end{abstract}

and intestinal reconnection surgery are discussed. PPN is not commonly used in this type of patients due to the short duration of the fast, although its use is common before surgery. However, postoperative use can be beneficial as well, and given the delicate postoperative state these patients are in, it is worth it (at least in these cases) to give them all the strength and tools available for a better recovery. PPN in the case discussed herein improved the patient's inflammatory marker levels in a shorter period.

(c) 2019 The Author(s). Published by S. Karger AG, Basel

\section{Introduction}

Patients who have undergone intestinal reconnection surgery after an oncological diagnosis in Morelia, Mexico, usually have indications for oral fasting for a duration of 3-5 days, according to their state and the presence of surgical complications. This, however, limits their necessary nutrient intake and in consequence promotes endogenous catabolism, thus elongating their hospital stay. Patients with gastrointestinal tumors are especially at risk for malnutrition complications relative to their diagnosis, hospitalization, and treatment (major surgery elicits a state of hypermetabolism for high-energy demands); factors that compromise the patient's ability to react to fasting periods and prognosis in general $[1,2]$.
Peripheral parenteral nutrition (PPN) is a powerful tool that can go a long way toward economizing protein energy, thus avoiding gluconeogenesis, muscular depletion, metabolic stress that further decreases anabolic hormones and increases catabolic ones, systemic inflammation, and sepsis [3].

Given that scarring is a protein-guided process (through hemostasis, inflammation, and tissue formation), a nitrogen balance in these patients is fundamental. A protein deficit can be associated with poor scarring and wound closing [4]. In this manner, inflammatory state, protein catabolism, and scarring process are associated and depend on each other.

Starvation during a metabolic stress process is different from a physiological fasting period. Surgery by itself is already a stress inducer in the organism that in turn promotes metabolic stress. 
Table 1. Screening Tool for CONUT

\begin{tabular}{lcccr}
\hline Parameter & \multicolumn{2}{l}{ Undernutrition degree } & \\
\cline { 2 - 5 } & normal & mild & moderate & severe \\
\hline Serum albumin, $\mathrm{g} / \mathrm{dL}$ & $\geq 3.5$ & $3.0-3.49$ & $2.5-2.99$ & $<2.5$ \\
Score & 0 & 2 & 4 & 6 \\
Total lymphocyte count, $/ \mathrm{mm}^{3}$ & $\geq 1,600$ & $1,200-1,599$ & $800-1,199$ & $<800$ \\
Score & 0 & 1 & 2 & 3 \\
Total cholesterol, $\mathrm{mg} / \mathrm{dL}$ & $\geq 180$ & $140-179$ & $100-139$ & $<100$ \\
Score & 0 & 1 & 2 & 3 \\
\hline
\end{tabular}

Biochemical indicators that reflect an individual's nutritional state, calculated by adding the scores of serum albumin, total lymphocyte count and total cholesterol. Modified by Ulibarri et al. [6]. CONUT score: Serum albumin + total lymphocyte count score + total cholesterol score. Undernutrition states: normal (score 0-1); mild (score 2-4); moderate (score 5-8); severe (score 9-12).

This is expected to be met by a strong metabolic response, which is fueled by a proper nutritional organic state for inflammation to stop spreading and healing to begin promptly [5].

To determine if a patient is at risk nutrition-wise, a clinical picture is yet to be established, which is why clinicians need to be able to interpret anthropometric, biochemical, clinical, and dietary data to assess the patient's nutritional state. The Controlling National Status score (CONUT) is the currently most accepted method for this determination, and its cutoff points are presented in Table 1 [6].

The aim of this case report is to determine if an inflammatory state derived from surgery shows improvement after hypocaloric PN (HPPN) administration (thereby lessening postsurgical risks), aside from the one in the patient's nutritional status.

\section{Case Presentation}

A 62-year-old female patient was admitted to a hospital in Morelia, Michoacán, with a diagnosis of ovarian cancer and gastrointestinal stromal tumor, with no previous oncological treatment, for a surgical plan for resection of the gastrointestinal stromal tumor, salpingo-oopherectomy, and entero-enteral anastomosis. The tumor was resected from the descending colon with the sigmoid colon as an anatomical limit, and the oncological diagnosis had been confirmed in a prior biopsy that led to the surgery herein discussed.

Upon arrival, a Penrose drain was placed during surgery for postsurgical fluid quantification to take into consideration together with oral diet tolerance for discharge. In addition, a consult for clinical nutrition was requested to assess nutritional state and administration of HPPN postoperatively. A Nutrition Care Process was initiated:

Anthropometric: the patient had a BMI of $23.7(45.8 \mathrm{~kg}, 1.39 \mathrm{~m})$, with a regular weight of $46 \mathrm{~kg}$ (ideal weight of $42.5 \mathrm{~kg}$, considering the proposed formula: $22 \times$ height $^{2}[\mathrm{~m}]$ ) [7].

Biochemical: upon admission, the patient's CONUT was scored at 9 , without considering the total lymphocyte count $(2.4 \mathrm{~g} / \mathrm{dL}$ albumin, $88.3 \mathrm{mg} / \mathrm{dL}$ cholesterol), indicating severe malnutrition. Furthermore, C-reactive protein of $18 \mathrm{mg} / \mathrm{dL}$ demonstrated sys- temic inflammation. On postoperative day 1, albumin and cholesterol levels dropped to $2 \mathrm{~g} / \mathrm{dL}$ and $39 \mathrm{mg} / \mathrm{dL}$, respectively, with an increase of CRP to $108 \mathrm{mg} / \mathrm{dL}$. In contrast, these same parameters at discharge showed the following: albumin of $3.2 \mathrm{~g} / \mathrm{dL}$, cholesterol of $125 \mathrm{mg} / \mathrm{dL}$, and a CRP of $48 \mathrm{mg} / \mathrm{dL}$, suggesting an important improvement of the nutritional state (from severe to mildto-moderate malnutrition), apparently, even though in this case we can presume that the improvement was in the patient's inflammatory state. The patient's biochemical evolution during hospital stay can be seen in Figure 1.

Clinical: vital signs during hospital stay were documented, where arterial pressure ranged from $102 / 68$ to $87 / 61 \mathrm{~mm} \mathrm{Hg}$ minimum, average temperature of $36.3^{\circ} \mathrm{C}$ (with only one spike to $37.6^{\circ} \mathrm{C}$ on day 3), and lowered peristalsis until normalization on day 4 . Drain quantification decreased gradually, starting on postoperative day 1 with 116-7 $\mathrm{mL}$ on day 6 (discharge).

Dietary: HPPN was administered with $50 \mathrm{~g}$ of amino acids, $150 \mathrm{~g}$ of dextrose, 1 multivitamin bottle, $1 / 2$ of an oligo element ampule, and $1 \mathrm{~g}$ of ascorbic acid. This resulted in a hypocaloric solution of $800 \mathrm{kcal}(17.46 \mathrm{kcal} / \mathrm{kg})$ and a positive nitrogen balance of +1.01 $\mathrm{g} / \mathrm{N}$ (taking into account a protein catabolism of $43.69 \mathrm{~g}$, obtained from a urine urea nitrogen of $6.99 \mathrm{~g}$ ). HPPN was administered since the first postoperative day, $1.5 \mathrm{~L}$ that contained the aforementioned nutrients and an osmolarity under $600 \mathrm{mOsm} / \mathrm{L}$, every $24 \mathrm{~h}$ for 3 days. Oral diet reintroduction was performed on postoperative day 4 with no difficulty, accomplishing adequate food digestion, absorption, and excretion without symptoms.

\section{Discussion and Conclusion}

The use of PPN in postoperative recovery of oncologic patients is rare because in most cases, short-term fasting is a safe option. However, the patient in the case presented here displayed a better scarring process with less inflammatory distress due to this approach. Data from a patient of a similar history not treated with PPN showed a decrease in albumin levels from 3.6 at admission to $2.4 \mathrm{~g} / \mathrm{dL}$ at discharge, and a cholesterol descent of 98.3 to 58.2 $\mathrm{mg} / \mathrm{dL}$. The mentioned parameters could have decreased abrupt-
62

Komp Nutr Diet 2021;1:61-63

DOI: $10.1159 / 000516241$ 


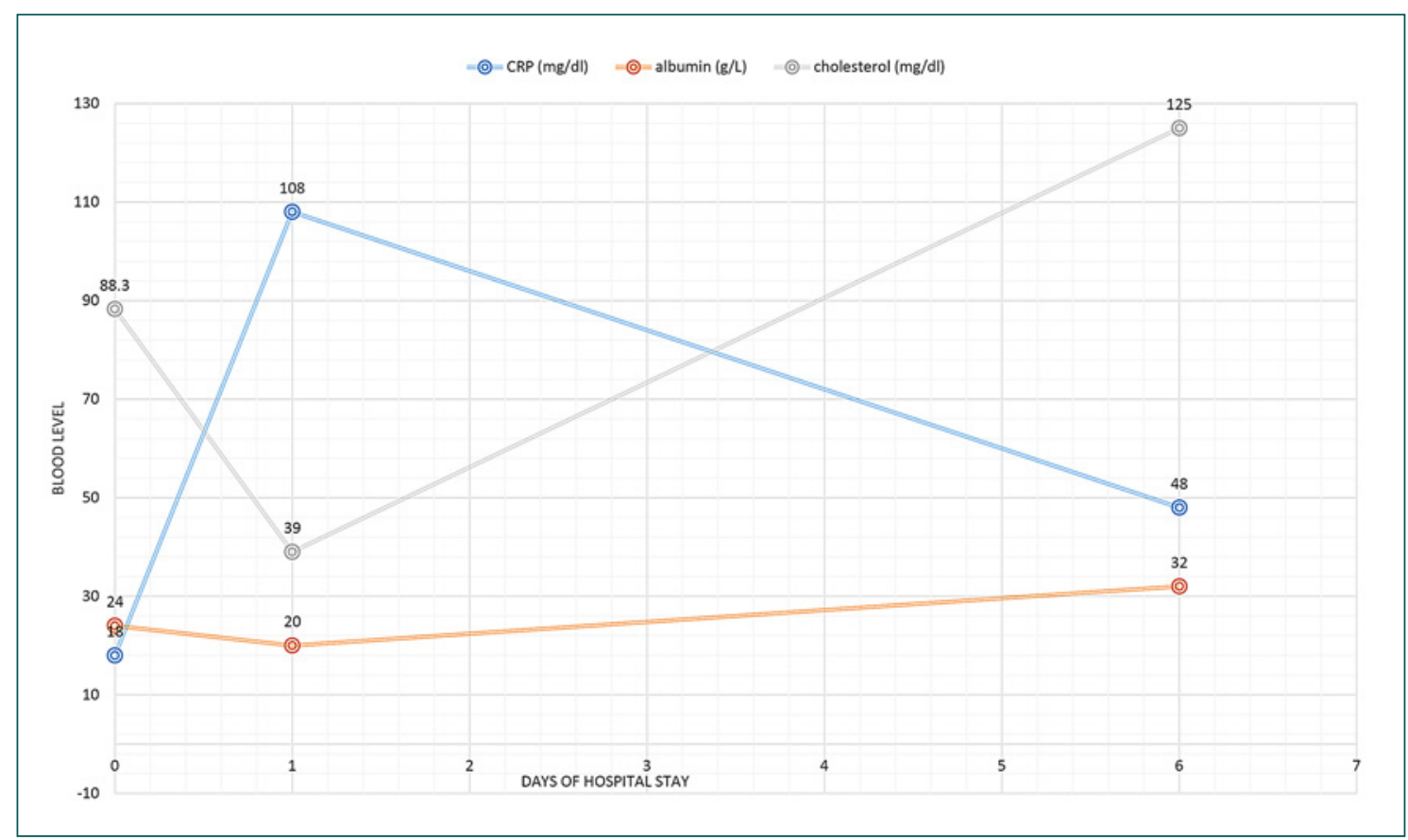

Fig. 1. Biochemical evolution during hospital stay. Day 0 is at admission, day 1 is after surgery, and day 6 is at discharge. Both albumin and cholesterol levels were below normal at admission, decreased postoperatively, and normalized towards discharge. CRP levels (inflammation marker) remained elevated throughout. However, they increased after the intervention and showed great improvement near discharge.

ly due to the surgery's aggressive component and not because of a nutritional lack. General guidelines describe PN as necessary in patients who are well nourished after 7 days, in patients at risk for malnutrition after 3-5 days, and in patients with total inability to ingest oral or enteral food immediately [8]. However, in this case (as in others alike), 3 days were enough to impact recovery, as others have documented as well. Pinzón et al. [9] determined that PN should initiate as soon as nutrient requirements are not met $>$ $60 \%$. Recent studies have also uncovered proof of the efficacy this therapeutic nutritional plan can have as a prophylactic measure for malnutrition and sepsis prevention [8].

Nutritional support is a more effective option when it is administered preoperatively, thus helping to avoid complications during postoperatively by strengthening the patient's nutritional status. However, in emergency cases where surgery is needed, a postoperative approach of $\mathrm{PN}$ is reasonable if we wish to decrease the risk of malnutrition and the complications it brings along.

HPPN was used in this case due to insufficient resources; however, PPN seems to be the better choice. PN as an immediate intervention in postoperative patients of intestinal reconnection is not the current standard of care; nonetheless, biochemical as well as clinical outcomes in the patient reported here show a clear benefit for reducing inflammation, and by proxy, malnutrition risk or anastomosis leak.

\section{Statement of Ethics}

The patient described in this case report gave written consent to use the relevant information for publication. The hospital where the case was studied also gave written permission to gather the information needed for the manuscript.

\section{Disclosure Statement}

The authors have no conflicts of interest to declare.

\section{Funding Sources}

This research did not receive any specific grant from funding agencies in the public, commercial, or not-for-profit sectors.

\section{Author Contributions}

Jesús Manuel De Aldecoa-Castillo carried out the acquisition of the patient data and helped draft the manuscript. Tamara Daniela Frydman was instrumental in the manuscript preparation. Daniela Rubio-Mendoza and Carlos Alvarado-Vargas supervised the case and patient data acquisition, and prepared the outline for the publication. Melchor Alpízar-Salazar edited the manuscript and gave approval to the final version.

\section{References}

References to this article are available at www.karger.com/doi/10.1159/ 000516241 . 